\title{
Teraherz generation from laser-driven ultrafast current propagation along wire target
}

\author{
H. B. Zhuo, ${ }^{1,2, *}$ S. J. Zhang, ${ }^{1}$ X. H. Li, ${ }^{1}$ H. Y. Zhou, ${ }^{1}$ X. Z. Li, ${ }^{1}$ \\ D. B. Zou, ${ }^{1, \dagger}$ H. C. Wu, ${ }^{3,2}$ Z. M. Sheng, ${ }^{4,5,2}$ and C. T. Zhou ${ }^{1,6}$ \\ ${ }^{1}$ College of Science, National University of Defense Technology, Changsha 410073, P. R. China \\ ${ }^{2}$ IFSA Collaborative Innovation Center, Shanghai Jiao Tong University, Shanghai 200240, P. R. China \\ ${ }^{3}$ Institute for Fusion Theory and Simulation and Department of Physics, \\ Zhejiang University, Hangzhou 310027, P. R. China \\ ${ }^{4}$ SUPA, Department of Physics, University of Strathclyde, Glasgow G4 ONG, United Kingdom \\ ${ }^{5}$ Key Laboratory for Laser Plasmas (MoE) and Department of Physics and Astronomy, \\ Shanghai Jiao Tong University, Shanghai 200240, P. R. China \\ ${ }^{6}$ Institute of Applied Physics and Computational Mathematics, Beijing 100094, P. R. China
}

(Dated: November 16, 2016)

\begin{abstract}
Intense coherent teraherz wave is emitted from laser accelerated high energetic electron beams collimated and guided along a limited length of wire. The radiated pattern shows much similar with transient radiation of energy from simple linear antennas driven by transient current pulse. Driven by $\sim 20 \mathrm{~mJ}$ ultra-short laser pulse with intensity $3 \times 10^{18} \mathrm{~W} / \mathrm{cm}^{2}$, teraherz wave with energy up to $\sim 0.15 \mathrm{~mJ}$, peak electric field $\sim 58 \mathrm{GV} / \mathrm{m}$, emission cone angle $\sim 30^{\circ}$, conversion efficiency $\sim 0.75 \%$ has been produced in simulations. An analytical description of the angular and frequency distribution of radiated energy is presented, which is show fairly agreement with particle-in-cell simulations.
\end{abstract}

PACS numbers: $52.25 \mathrm{Fi}, 52.40 . \mathrm{Mj}, 52.27 . \mathrm{Kk}$

Interaction of high-energy and high-power laser pulses with solid targets is accompanied with the generation of a significant number of energetic electrons, producing many secondary effects. The bright $\mathrm{x}$ and $\gamma$-ray emission [1], intense ion acceleration [2, 3], and many other effects [4-6] have been intensively studied and have been used in many applications. Furthermore, several efforts have been put on the generation of intense electromagnetic pulses during and after the laser plasma interaction, especially with the frequency ranged in teraherz $(\mathrm{THz})$ band [7-11]. It is because that plasma would overcome the limitations associated with damage in nonlinear crystal-based terahertz sources. Terahertz sources utilizing laser plasmas are a promising technology for creating table-top equipment to generate extremely strong terahertz pulses with energies approaching the millijoule level. More recently, efficient terahertz pulse generation has been demonstrated in several experiments by irradiating a thin metal foil with an ultrashort intense laser pulse $[12,13]$. Correspondingly two major mechanisms have been proposed. One is coherent transition radiation [12], which take place when laser accelerated electrons reaching the rear side of the target and transporting across the interface between the rear target and vacuum. The other is target normal sheath radiation [13], which emit from the electrons deaccelerated by an intense sheath field formed on the rear surface. In both scenarios, the radiation are closely related to the characteristics of these electron sources and its transient dynamic. The achieved radiation power was posed significant constraints by the large angular divergence of the electron beam, which quickly reducing the electrons density in a short distance.
Some remarkable phenomena have been observed in relativistic laser interactions with wire targets [14-18]. Collimation and guidance of fast electrons with energies of a few hundred $\mathrm{keV}$ or $\mathrm{MeV}$ has been demonstrated using wire targets $[14,17,18]$. Field propagation at the speed of light along a wire has been observed by laserdriven proton radiography and induced transient current with a peak magnitude up to the order of $10^{4} \mathrm{~A}$ [16]. It has been suggested the surface radial electric field or balance of the surface radial electric field and the azimuthal magnetic field force results in fine collimation effect of electrons $[14,19,20]$. In this paper, we introduce a different approach to produce ultra-strong single cycle $\mathrm{THz}$ sources from interaction of intense lasers with solid wire target. As illustrated in Fig. 1(a), we consider an intense laser pulse incident obliquely onto the center of the wire target and accelerate a large number of energetic electrons. As most electrons are effectively collimated and guided along the wire, a huge transient currents could be formed on the wire surface in responding to moving fields of electrons. Consider the temporal profile of transient current roughly follows the laser pulse (typically an order of a few fs or more for TW laser system), thus the Fourier transform of the pulse envelope yields a carrier frequency $\sim 10^{13} \mathrm{~Hz}$. It is naturally a coherent driven source in the desired $\mathrm{THz}$ frequency range, which drives the wire as a current-carrying line antenna in turn to emit electromagnetic radiations.

To demonstrate the novel features, a series of twodimensional simulations were carried out by using particle-in-cell (PIC) code PDLPICC2D [21, 22]. In the simulations, a p-polarized laser pulse perpendicularly incident from the left boundary onto the center of 


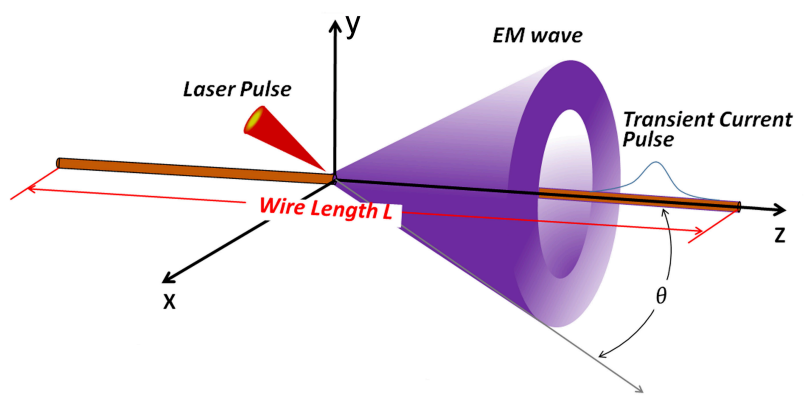

FIG. 1: (color online.) Principle illustration of the scheme of the $\mathrm{THz}$ generation. Transient current formed at center of wire target is propagating along the wire to the right end of the target. The wire is directed along $\mathrm{z}$ axis and $\theta$ is the observation angle.

the wire target, which is located at the center of the simulation box with an oblique angle $30^{\circ}$ in respect to the laser axis. The laser intensity is set as $3.08 \times 10^{18}$ $\mathrm{W} / \mathrm{cm}^{2}$, corresponding to the normalized vector potential $a_{0}=e E / m_{e} \omega_{0} c=1.5$, where $m_{e}$ is electron mass, $\mathrm{c}$ is the speed of light, and $\omega_{0}$ is laser frequency. The intensity profile is Gaussian in the y direction with a focus spot size $\mathrm{w}_{0}$ of $6 \lambda_{0}$ (full width at half maximum). The time profile is $e^{-t^{2} / 2 \tau_{0}^{2}}$ with $\tau_{0}=3 T_{0}$, where $T_{0}$ is laser period. The length of wire target is $L=30 \lambda_{0}$ and the thickness is $D=2.0 \lambda_{0}$. The simulation box is $100 \lambda_{0} \times 100 \lambda_{0}$ with a spatial resolution up to 100 cells per wavelength. Each cell contains 100 ions and 100 electrons. The target is assumed as fully ionized and the electron density is 10 times critical density $\left(n_{c}\right)$, which corresponds to $1.12 \times 10^{21} \mathrm{~cm}^{-3}$ for laser wavelength $\lambda_{0}=1.0 \mu \mathrm{m}$. In order to resolve the Debye length of plasma in our simulations, the initial electron temperature is $1 \mathrm{keV}$ and ions are fixed.

The distinctive pattern of the radiation emitted from laser driven wire target was shown in Fig. 2. Two spherical waves front, one centered at the middle of the wire and other centered at right end of the wire, were clearly distinguished. The first spherical wave was formed when the laser pulse reach at the center of the target and energetic electrons was accelerated by the laser force. The second spherical wave is generated when hot electrons propagating stably long the wire reach the termination on the right. After that two spherical wave was escaping from the individual source and traveling outward at the speed of light. According to the direction and magnitude of the field components, the Poynting vectors can be inferred to be predominantly normally over a spherical wavefront. The intensity of radiation is not distributed uniformly over the spherical wavefront, but obviously dependent on the emission angle. All this characteristic are much similar to typical radiation from a linear antenna driven by transient current pulse [24].

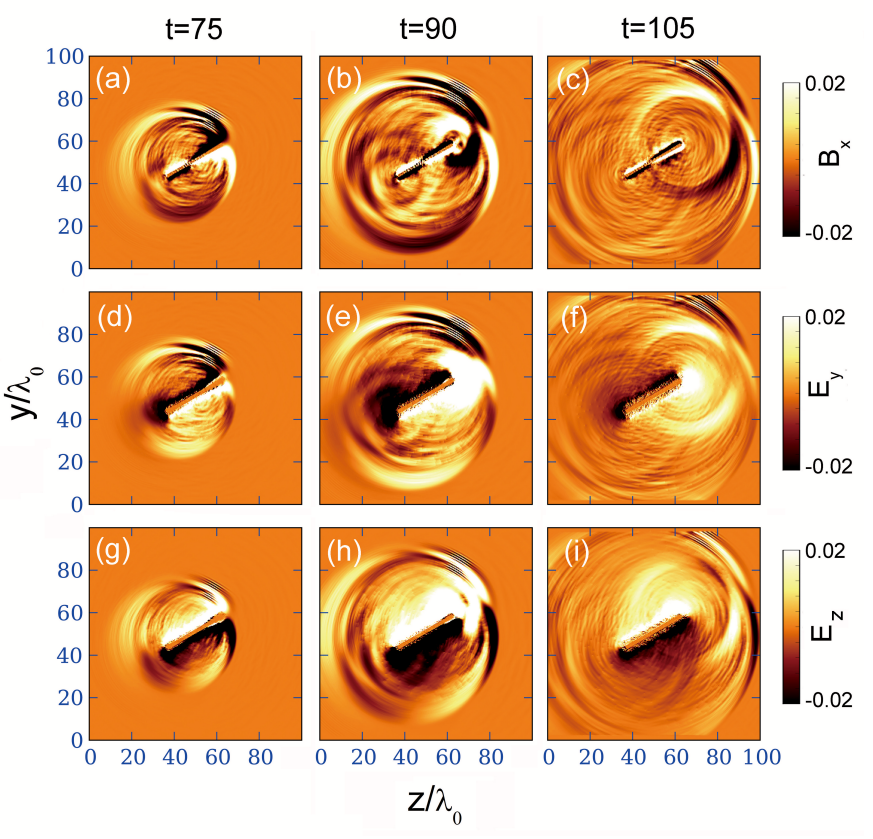

FIG. 2: (Color online.) Snapshots of the radiation field components $B_{x}, E_{y}$ and $E_{z}$ at three different times respectively. $\mathbf{E}$ and $\mathbf{B}$ are normalized by $m_{e} \omega_{0} c / e$.

On the condition that the intense laser pulses oblique incident on the wire target with large angle, the electron acceleration mechanism is much close to that described in $[19,20]$. As shown in Fig. 3(a,b), except few electrons can escape from the target, most energetic electrons are propagating along the wire with good collimation. The guiding effect could also be inferred from evolvement of the surface field as shown in Fig. 3(c-f). The intense radial electric field $E_{y}$, induced by the charge-separation field of the escaped high-density $\mathrm{MeV}$ electrons, tends to pull the electrons back to the surface. However, an intense azimuthal magnetic field $B_{x}$ of the order of a few hundred MG is also created, and it tends to push the electrons away from the surface. A balance of the electric force $e E_{y}$ and magnetic force $(e / c) \boldsymbol{v}_{e} \times \boldsymbol{B}$ results in the collimation and confinement of the fast electrons, which moves laterally at approximately speed of light. The dynamic behavior of the electron beams play important role in the radiation production. Simulations with other incident angles were also carried out. It is found that the radiation strength decrease seriously for small incident angle because electrons collimation do not take place effectively.

On the base of Ampere's law, the currents can be roughly estimated by the surface magnetic field strength as $I \approx \pi B_{x} D / \mu_{0}$. It is $\sim 11 \mathrm{KA}$ for $B_{x} \approx 0.2 m_{e} \omega_{0} c / e \sim$ 2200 Tesla (obtained in Fig. 3). Integration current over pulse length reveals the net number of electrons moving along the wire to be $\sim 1.72 \times 10^{9}$. As a comparison, the total number of hot electrons accelerated are also esti- 


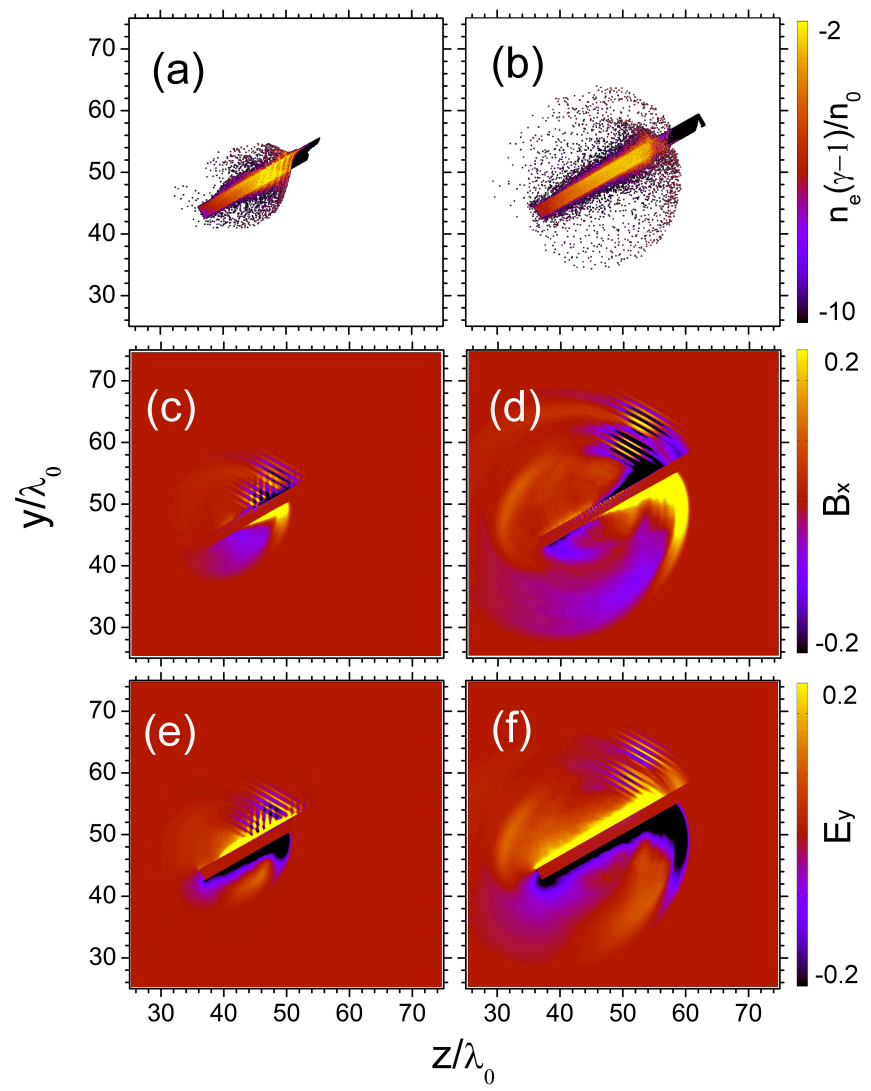

FIG. 3: (Color online.) The electron kinetic energy density (a,b), the self-generated surface magnetic field $B_{x}(\mathrm{c}, \mathrm{d})$, and the electric field $E_{y}(\mathrm{c}, \mathrm{d})$ at $t=55$ (left column), 65 laser periods (right column), respectively. $\mathbf{E}$ and $\mathbf{B}$ are normalized by $m_{e} \omega_{0} c / e$.

mated by energy balance relation $N_{\text {total }}=f E\left(k_{B} T_{h}\right)^{-1}$, where $f$ is the fraction of laser energy absorbed by hot electrons, $E \sim 20 \mathrm{~mJ}$ is the pulse energy, and $k_{B} T_{h} \sim 0.2$ $\mathrm{MeV}$ is the hot electron temperature predicted by Ponderomotive scaling [23]. Taking $f \sim 10 \%$ from the simulations, total laser energy is estimated to accelerate some $6 \times 10^{10}$ hot electrons at its focus. This contrasts starkly with the number of electrons contributed to the transient current priorly estimated. That means only fastest electrons, typically those with an energy larger than the temperature of the Maxwellian distribution, contribute to the transient current. The reminder will return to the wire under spacecharge force and neutralized by the wire plasma.

In the following, the line antenna model proposed by Simth [24] was used to analysis the angular and frequency distribution of the radiation. For simplicity, we assume a transient current with gaussian profile was formed at $z=0$, propagating along the wire to the end of the target with speed of light $c$. Further neglecting the temporal variance of the current profile during short propagation process, the transient currents induced on the wire can be simply described as $I(z, t)=I_{s}(t-z / c)$, where $I_{s}(t)=I_{0} e^{-t^{2} / 2 \tau_{0}^{2}}$ is current time profile, and $I_{0}$ is peak current intensity. In the limit as $r / L \rightarrow \infty$, where $r$ is the distance to the field point, the radiated electric field of the moving current source is given by the Smith's model as [24]

$$
\begin{aligned}
& \overrightarrow{\mathbf{E}}(\overrightarrow{\mathbf{r}}, t)=\frac{\mu_{0} c \sin \theta}{4 \pi r(1-\cos \theta)} \\
& \quad\left\{I_{s}(t-r / c)-I_{s}\left(t-r / c-\tau_{d}\right)\right\} \hat{\theta}
\end{aligned}
$$

where $\theta$ is observation angle with respect to the wire direction, and $\tau_{d}=\frac{L}{2 c}(1-\cos \theta)$ is the difference of the retarded time between the source and terminal. Based on (1), the spectral intensity of the radiation can be obtained as

$$
\frac{d^{2} I}{d \Omega d \omega}=\frac{\mu_{0} c I_{0}^{2} \tau_{0}^{2} \sin ^{2} \theta}{4 \pi(1-\cos \theta)^{2}} \sin ^{2} \frac{\omega \tau_{d}}{2} e^{-\tau_{0}^{2} \omega^{2}}
$$

After integrating (2) over the frequency, we obtain the radiation energy into the solid angle as

$$
\frac{d W}{d \Omega}=\frac{\mu_{0} c I_{0}^{2} \tau_{0} \sin ^{2} \theta}{16 \sqrt{\pi}(1-\cos \theta)^{2}}\left(1-e^{-\tau_{d}^{2} / 4 \tau_{0}^{2}}\right)
$$

Further integrating Eq. (3) over the solid angle $\Omega$, the total energy radiated from the wire is obtained as

$$
W=\frac{\mu_{0} c I_{0}^{2} \tau_{0}}{4 \sqrt{\pi}}\left[\gamma-2+\ln \alpha^{2}+\frac{\sqrt{\pi}}{\alpha} \operatorname{Erf}(\alpha)+E_{1}\left(\alpha^{2}\right)\right]
$$

Here, $\alpha=\frac{L}{2 c \tau_{0}}, \gamma=0.57721$ is Euler's constant, Erf is the error function, and $\mathrm{E}_{1}$ is exponential integrals.

The above model fairly reproduced the radiation pattern observed in the simulation. As shown in Fig. 4(a), the radiated electric field $E_{\theta}$ at $\mathrm{t}=50 T_{0}$, calculated by Eq. (1) with the simulation parameters, show an almost similar structure as shown in Fig. 2(f). According to the expression on the right-hand side of Eq. (1), the first spherical wave of larger radius is produced when the current pulse formed on the center. Another term is produced when the current pulse is absorbed by the terminal. These two wavefronts, with the opposite electric field direction and the different retarded time $\tau_{d}$, is overlapped in far-region. It leads to the power of radiation not distributed uniformly over the spherical wavefront, but obviously dependent on the observation angle. The spectra calculated from Eq. (2) is shown in Fig. 4(b). One can see that no emission is observed at $\theta=0^{\circ}$. The central peak is located at $\theta=57^{\circ}$, with the center frequency is about $\omega \sim 0.8 / \tau_{0}=16 \mathrm{THz}$. It roughly equals to the carrier frequency of driven laser pulse envelope. The whole spectra are extended from 0 to $3 / \tau_{0}$ for small observation angle. The spectrum peak is shifted to lower frequencies with the bandwidth reducing dramatically with the observation angle increasing, . 
Fig. 4(c) shows the angular distribution of radiated energy for different wire length calculated by Eq. (3). The time-integration of the radiation flux cross the observation plane in simulations is also plotted for comparison. Good fitness with the model prediction of $\mathrm{L}=30 \mu \mathrm{m}$ further prove the reliability of our model. As the wire length reduce to $\mathrm{L}=6 \mu \mathrm{m}$, one can see that the peak radiation direction shift to the vertical direction of the wire with the intensity decrease. On the contrary, the radiation is more enhanced and concentrated into a hollow forward cone by increasing the wire length, as shown by curve for $\mathrm{L}=800 \mu \mathrm{m}$. It is similar to the characteristic of radiation from a moving point charge when the acceleration is parallel to the velocity, whose angular distribution and intensity is dependent on the kinetic energy of electron.

Fig. 4(d) shows the temporal profiles of the forward flux $F^{+}=\left(E_{y}-c B_{x}\right) / 2$ cross the observation plane. It was recorded at $\theta=30^{\circ}$ in our simulation. The whole signal presents as a single cycle pulse. The second half-cycle shows disordered in compared with the first half-cycle. It was attributed to the variance of profile during electron beams moving along the wire target. As a result, red shift of emission frequency from the model prediction $\omega=0.05 \omega_{0}$ to simulation results $\omega=0.04 \omega_{0}$ could
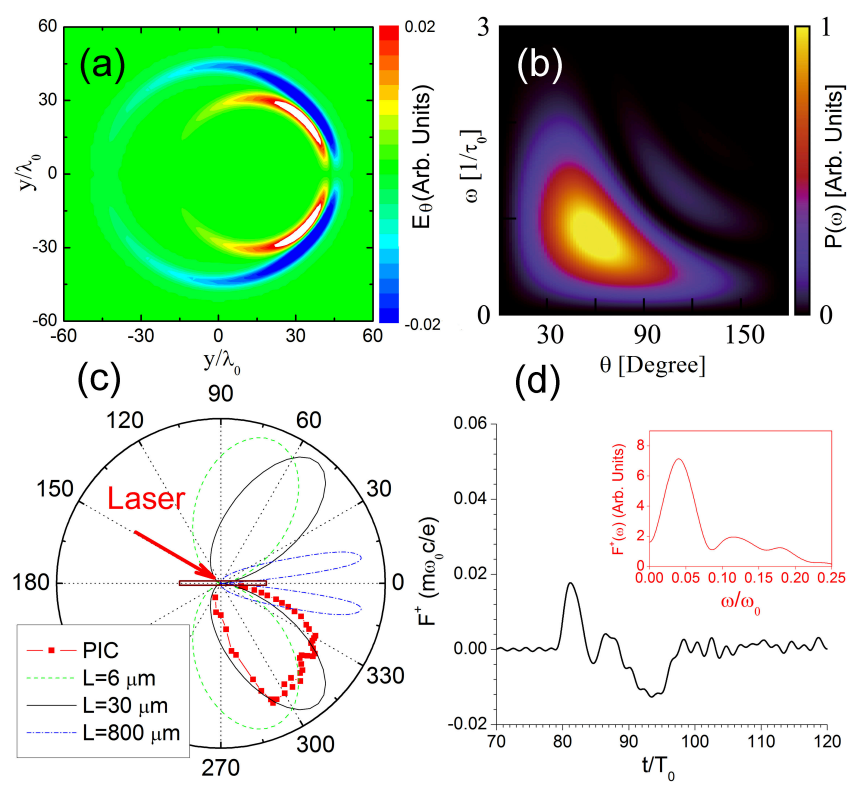

FIG. 4: (color online.) (a) Model predicted radiation pattern of electric field $E_{\theta}$, (b) emission spectra as a function of the observation angles $\theta$, and (c) the angular distribution of the radiation power for different wire lengths. The solid squares represent the integration of flux over time obtained from simulation fitted for $L=25 \mu \mathrm{m}$. The curves for $L=6 \mu \mathrm{m}$ and $L=800 \mu \mathrm{m}$ have been magnified by a factor of 3 and $1 / 80$, respectively. (d) The temporal profile of radiation flux cross the observation plane $\left(\theta=30^{\circ}\right)$ and the radiated spectra (in inset) obtained from simulation.

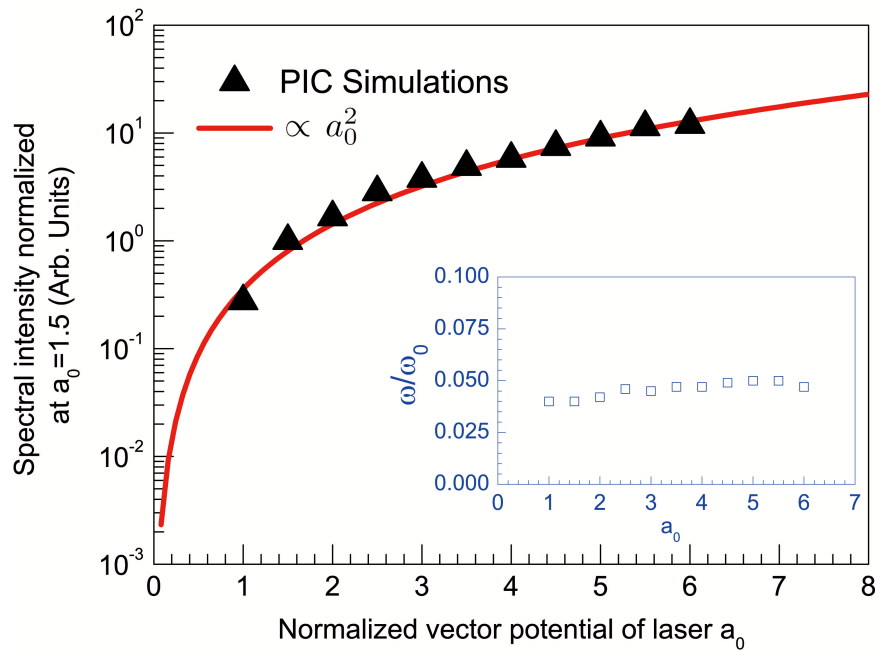

FIG. 5: (Color online.) Parametric dependence of the radiation intensity $\left(\theta=30^{\circ}\right)$ and the center frequency (in inset) as a function of the laser intensity. Solid line is from model prediction, and filled triangles and hollow squares represent PIC simulation results.

be distinguished (shown in inset). The peak amplitude of flux is over $0.018 m_{e} \omega_{0} c / e$, corresponding to electric field strength over $58 \mathrm{GV} / \mathrm{m}$. Integrated over the emission cone angle $\Omega \sim 30^{\circ}$ and duration $\sim 20 T_{0}$ (i.e., 66 fs), the whole radiated energy is estimated about $\sim 0.15$ $\mathrm{mJ}$. The conversion efficiency from laser to radiation is over $0.75 \%$. Taking this radiated energy into Eq. (4), the driven current derived is $\sim 10 \mathrm{KA}$, which is fairly consistent with the prior estimation.

The dependence of the radiated power on the laser intensity can be easily derived. Based on Eq. (3), the radiated power is proportional to the square of the current intensity, which is determined by the laser intensity and the conversion efficiency from laser to fast electrons. On current condition that the ponderomotive force effect is dominant for hot electron generation, the current is proportional to the total number of hot electrons accelerated. Thus the scaling of the radiated power could be simply described as $P \propto a_{0}^{2}$. It is fitted well with the simulation results as shown in Fig. 5. Moreover, the center frequency of radiation is almost independent with the laser intensities (shown in inset). It is consistent with our assumption that only fastest hot electrons really contributes to the final radiation.

From the viewpoint of potential applications, it is useful to efficiently transmit such intense radiation from the laser-produced source to a long distance destination. According to expression in Eq. (3), in the limit as $L \rightarrow \infty$, the optimal radiated angle $\theta \rightarrow 0$. It implied that whole radiation would be transfer as a surface wave and propagating along the wire lines. The ultimate limit for surface wave intensity is attributed to resistive heating by a transient current and ionization of the wire surface by a 
propagating surface wave. In fact, several theoretic and experimental results have proved that the metal wires can also be served as excellent low loss and low dispersion waveguide for Sommerfeld waves in the gigahertz to terahertz frequency range $[25,26]$.

In summary, we propose a simple route to produce the intense coherent $\mathrm{THz}$ wave by obliquely incident the intense laser pulse onto the wire target. While fast electrons are confined and flow along the wire line, the wire is equivalent to a linear antenna and in turn radiate electromagnetic energy. The intense $\mathrm{THz}$ wave with the electric field more than $58 \mathrm{GV} / \mathrm{m}$, conversion efficiency from laser to radiation up to $0.75 \%$, has been obtained in simulations. An analytical description of the angular and frequency distribution of radiated energy is present, which is show fairly agreement with the 2D PIC simulation results. Our proposed scheme is offering a route to produce ultra-strong $\mathrm{THz}$ sources for applications such as pump-probe high field $\mathrm{THz}$ irradiation experiments. Moreover, it provides a unique way to understand the long distance transport of a large flux of electron beam along the wire target, which is potentially important for high energy density physics driven by ultrashort high intensity lasers. This technique of $\mathrm{THz}$ generation works well only if the driven laser pulse has a very high contrast and vary shot duration. Because of the prepulse or long pulses can lead to strongly plasma expanding and destruction of wire structures. Fortunately, the techniques such as the plasma mirror to achieve ultrahigh pulseto-prepulse contrast ratios now offer the opportunity to carry out such experimental studies at very high intensity. The conditions of vary short laser pulse used in our simulations are easily met by current laser facility, such as the chirped pulse amplification laser at the Center of Ultrafast Optical Science (CUOS) [27].

This work is supported by the National Basic Research Program of China (2013CBA01504), the Science Challenge Program, the National Natural Science Foundation of China (11475259, 11175253 and 91230205), and the Research Program of NUDT. We would like to thank the Guangzhou National Supercomputing Center for providing their computing facilities.

* Electronic address: hongbin.zhuo@gmail.com

Electronic address: debinzou@126.com

[1] K. W. D. Ledingham, I. Spencer, T. McCanny, R. P. Singhal, M. I. K. Santala, E. Clark, I. Watts, F. N. Beg, M. Zepf, K. Krushelnick, M. Tatarakis, A. E. Dangor, P. A. Norreys, R. Allott, D. Neely, R. J. Clark, A. C. Machacek, J. S. Wark, A. J. Cresswell, D. C. W. Sanderson, and J. Magill, Phys. Rev. Lett. 84, 899 (2000)

[2] E. L. Clark, K. Krushelnick, J. R. Davies, M. Zepf, M. Tatarakis, F. N. Beg, A. Machacek, P. A. Norreys, M. I. K. Santala, I. Watts, and A. E. Dangor, Phys. Rev. Lett.
84, $670(2000)$

[3] H. B. Zhuo, Z. L. Chen, W. Yu, Z. M. Sheng, M. Y. Yu, Z. Jin, and R. Kodama, Phys. Rev. Lett. 105, 065003 (2010).

[4] Z. Jin, Z. L. Chen, H. B. Zhuo, A. Kon, M. Nakatsutsumi, H. B. Wang, B. H. Zhang, Y. Q. Gu, Y. C. Wu, B. Zhu, L. Wang, M. Y. Yu, Z. M. Sheng, and R. Kodama, Phys. Rev. Lett. 107, 265003 (2011).

[5] S. J. Zhang, H. B. Zhuo, D. B. Zou, L. F. Gan, H. Y. Zhou, X. Z. Li, M. Y. Yu, and W. Yu. Phys. Rev. E 93, 053206 (2016)

[6] H. B. Zhuo, Z. Jin, M. Y. Yu, Z. M. Sheng, H. Xu, Y. Y. Ma, Y. Yin, F. Q. Shao, W. M. Zhou, and R. Kodama, Phys. Plasmas. 19, 043108 (2012).

[7] H. Hamster, A. Sullivan, S. Gordon, W. White, and R. W. Falcone, Phys. Rev. Lett. 71, 2725 (1993).

[8] H. Hamster, A. Sullivan, S. Gordon, R.W. Fal- cone, Phys. Rev. E 49, 671 (1994).

[9] A. Sagisaka, H. Daido, S. Nashima, S. Orimo, K. Ogura, M. Mori, A. Yogo, J. Ma, I. Daito, A. S. Pirozhkov, S. V. Bulanov, T. Z. Esirkepov, K. Shimizu, and H. Hosoda, et al., Appl. Phys. B 90, 373 (2008).

[10] Y. T. Li et al., Appl. Phys. Lett. 100, 254101 (2012).

[11] G. Q. Liao et al., Phys. Rev. Lett. 114, 255001 (2015).

[12] G. Q. Liao, Y. T. Li, T. H. Zhang, H. Liu, X. L. Ge, S. Yang, W. Q. Wei, X. H. Yuan, B. J. Zhu, Z. Zhang, W. M. Wang, Z. M. Sheng, L. M. Chen, X. Lu, J. L. M, X. Wang, and J. Zhang, Phys. Rev. Lett. 116, 205003 (2016)

[13] A. Gopal, S. Herzer, A. Schmidt, P. Singh, A. Reinhard, W. Ziegler, D. Brommel, A. Karmakar, P. Gibbon, U. Dillner, T. May, H-G. Meyer, and G. G. Paulus, Phys. Rev. Lett. 111, 074802 (2013)

[14] R. Kodama, Y. Sentoku, Z. L. Chen, G. R. Kumar, S. P. Hatchett, Y. Toyama, T. E. Cowan, R. R Freeman, J. Fuchs, Y. Izawa, M. H. Key, Y. Kitagawa, K. Kondo, T. Matsuoka, H. Nakamura, M. Nakatsutsumi, P. A. Norreys, T. Norimatsu, R. A. Snavely, R. B. Stephens, M. Tampo, K. A. Tanaka, and T. Yabuuchi, Nature 432, 1005 (2004).

[15] F. N. Beg et al., Phys. Rev. Lett. 92, 095001 (2004)

[16] K. Quinn et al., Phys. Rev. Lett. 102, 194801 (2009)

[17] S. Tokita, K. Otani, T. Nishoji, S. Inoue, M. Hashida, and S. Sakabe, Phys. Rev. Lett. 106, 255001 (2011).

[18] H. Nakajima, S. Tokita, S. Inoue, M. Hashida, and S. Sakabe, Phys. Rev. Lett. 110, 155001 (2013).

[19] T. Nakamura, S. Kato, H. Nagatomo, and K. Mima, Phys. Rev. lett 93, 265002 (2004).

[20] Y. T. Li, X. H. Yuan, M. H. Xu, Z. Y. Zheng, Z. M. Sheng, M. Chen, Y. Y. Ma, W. X. Liang, Q. Z. Yu, Y. Zhang, F. Liu, Z. H.Wang, Z. Y.Wei, W. Zhao, Z. Jin, and J. Zhang, Phys. Rev. Lett. 96, 165003 (2006).

[21] H. B. Zhuo, W. Yu, M. Y. Yu, H. Xu, X. Wang, B. F. Shen, Z. M. Sheng, and J. Zhang, Phys. Rev. E 79, 015401(R) (2009).

[22] H. B. Zhuo, Z. L. Chen, Z. M. Sheng, M. Chen, T. Yabuuchi, M. Tampo, M. Y. Yu, X. H. Yang, C. T. Zhou, K. A. Tanaka, J. Zhang, and R. Kodama, Phys. Rev. Lett. 112, 215003 (2014).

[23] S. C. Wilks and W. L. Kruer, IEEE J. Quantum Electron 33, 1954 (1997).

[24] G. S. Smith and Thorsten W. Hertel, IEEE Anteenas and Propagation Magzine. 43, 49 (2001).

[25] G. Goubau, J. App. Phys. 21, 1119 (1950).

[26] K. Wang and D. M. Mittleman, Phys. Rev. Lett. 96, 
157401 (2006).

[27] G. I. Dudnikova, V. Yu. Bychenkov, A. Maksimchuk, G. Mourou, J. Nees, S. G. Bochkarev, and V. A. Vshivkov,
Phys. Rev. E 67, 026416 (2003). 